\title{
Migrations en Afrique australe
}

Levier de la Renaissance ou facteur d'inégalités?

Migration in Southern Africa. Leverage of African Renaissance or Factor of Inequalities?

\section{Aurelia Wa Kabwe-Segatti}

\section{OpenEdition \\ Journals}

Édition électronique

URL : http://journals.openedition.org/transcontinentales/452

DOI : 10.4000/transcontinentales.452

ISBN : 978-2-8218-1408-0

ISSN : 1775-397X

Éditeur

Editions de la maison des sciences de l'homme

\section{Édition imprimée}

Date de publication : 30 juin 2006

Pagination : 77-99

ISBN : 2200-92169-1

ISSN : 1950-1684

\section{Référence électronique}

Aurelia Wa Kabwe-Segatti, « Migrations en Afrique australe », Transcontinentales [En ligne], 2 | 2006, mis en ligne le 30 septembre 2012, consulté le 07 septembre 2020. URL : http://

journals.openedition.org/transcontinentales/452 ; DOI : https://doi.org/10.4000/transcontinentales. 452

Ce document a été généré automatiquement le 7 septembre 2020

Tous droits réservés 


\title{
Migrations en Afrique australe
}

\author{
Levier de la Renaissance ou facteur d'inégalités? \\ Migration in Southern Africa. Leverage of African Renaissance or Factor of \\ Inequalities?
}

Aurelia Wa Kabwe-Segatti

Bien que les migrations aient modelé les espaces, les identités et la structure socioéconomique de l'Afrique australe depuis deux siècles, on observe depuis la fin de l'apartheid une transformation sans précédent tant du volume que de la diversité des populations de migrants qui entrent en Afrique du Sud et, dans une moindre mesure, chez ses deux voisins relativement nantis que sont le Botswana et la Namibie. On pourrait penser que le phénomène actuel ne relève que d'une évolution de ces migrations séculaires, largement étudiées ${ }^{1}$. Pourtant, force est de constater que, tant les statistiques officielles ${ }^{2}$ que les corpus quantitatif ${ }^{3}$ et qualitatif des sciences sociales sur la question font apparaitre une modification en profondeur du volume et de la nature des flux de migrants depuis le début des années 1990. C'est cette mutation qu'il s'agit de mieux comprendre ici. Les migrations temporaires des secteurs minier et agricole ont en effet subi des changements récents qui leur sont propres (sur lesquels nous reviendrons), mais de nouvelles formes de migration sont aussi apparues, animées par des populations originaires de l'ensemble du continent et du reste du monde. Les durées de séjour de ces migrants, leurs motivations, les facteurs socio-économiques et politiques qui sous-tendent leur mobilité, enfin l'impact de celle-ci tant sur les pays d'accueil (Afrique du Sud, Botswana, Namibie) que sur leurs pays d'origine sont des données nouvelles qui commencent, depuis moins de dix ans, à être systématiquement explorées par la recherche en sciences sociales.

2 Cette nouvelle configuration prend une importance accrue si l'on pense au rôle continental qu'entend jouer l'Afrique du Sud depuis son retour dans la communauté internationale. Intégrant la Southern African Development Community (SADC) en 1996, l'Afrique du Sud, particulièrement sous la présidence de Thabo Mbeki (1999 à ce jour), a affiché des ambitions continentales fortes, sur le plan politico-diplomatique en direction du Burundi, de la République démocratique du Congo (RDC) et de la Côte d'Ivoire, comme sur le plan économique avec l'expansionnisme de son secteur privé, 
donné aujourd'hui comme le premier investisseur africain sur le continent africain ${ }^{4}$. L'article de Garth le Pere dans ce numéro en dresse un bilan nuancé. Le rôle moteur de Pretoria dans la réforme de l'Organisation de l'unité africaine, devenue depuis Union africaine, et dans l'initiative du New Economic Partnership for Africa's Development (NEPAD), lui donne aujourd'hui une place de leader - contesté sans grande crédibilité par le Nigeria ou la Libye - du continent africain. Dans ce contexte, que peut-on dire du rôle joué par ces nouvelles migrations, cette forme de "mondialisation par le bas ${ }^{5}$ ", dans la relation en devenir entre une Afrique australe privilégiée mais fragile et le reste du continent?

3 Avant d'envisager les transformations majeures du cadre migratoire dans l'Afrique australe post-apartheid, il est essentiel de situer les enjeux économiques qui structurent aujourd'hui la réflexion sur la dialectique migration-développement, ne serait-ce que pour mieux comprendre ensuite les choix politiques qui contraignent les gouvernements de ces nouveaux eldorados du Sud que sont devenus l'Afrique du Sud, le Botswana et la Namibie. Terre d'exil, de transit et d'installation, la pointe sud de l'Afrique australe prend aujourd'hui pleinement conscience des défis liés à son entrée dans le monde. Face à un cosmopolitisme tour à tour revendiqué et honni, les gouvernements de ces trois pays progressent, selon des modalités différenciées, dans la voie de constructions nationales qui ne pourront faire l'économie d'une réflexion plus large sur l'étranger.

\section{Migration et développement}

4 Sans prétendre épuiser cette thématique, il semble essentiel de revenir sur la question très débattue de la relation entre migration et développement. Les rapports récents de la Banque mondiale et de la Commission mondiale sur les migrations internationales ${ }^{6}$ sur le volume des envois d'argent des migrants dans le monde ont relancé une interrogation ancienne sur les effets d'entraînement que pourraient avoir les migrations sur le développement des sociétés d'origine. Ce débat aura, semble-t-il, bien du mal à être tranché, tant les économistes conviennent de la difficulté à «mesurer » aussi bien l'impact direct de ces envois d'argent, que celui d'autres transferts (acquisitions et constructions de logement, apports de biens de consommation), sans même parler de l'impossibilité de mesurer ces effets-là en regard de la perte que constitue le départ de la personne qualifiée sur l'économie d'origine.

Dans une enquête réalisée en $1996^{7}$, Maurice Schiff, du département d'économie internationale de la Banque mondiale, concluait que « [la] question de la substitution ou de la complémentarité entre commerce et migration ne présente aucune réponse définitive. On peut dire la même chose de l'impact de la migration sur les systèmes d'aide sociale. " Appliquant différents modèles économétriques, Schiff parvient à la conclusion que, quel que soit le degré d'internalisation des coûts marginaux de la migration, le Sud gagnerait à libéraliser l'accès à ses marchés et le Nord à imposer une taxe sur l'immigration. On le voit, Schiff peine à expliquer les implications macroéconomiques de la migration, tant les paramètres sont nombreux et difficilement modélisables. Au risque d'aboutir à des conclusions idéologiquement situées (toute chose égale par ailleurs, l'ouverture des marchés serait forcément positive) dont la réalisation en termes de politique publique reste très incertaine (on comprend mal comment appliquer une taxe sur l'immigration irrégulière par exemple). En outre, les 
migrations forcées, moins systémiques, sont écartées des modèles. Cette enquête démontre toutefois que, contrairement aux certitudes tirées de références devenues classiques ${ }^{8}$, il est vain de lier mécaniquement commerce et main-d'œuvre migrante, capital et facteur de production. Un rapport récent de la Banque mondiale semble confirmer aujourd'hui que les envois ou remises de fonds réalisés par les migrants dans le monde dépassent le niveau de l'aide publique au développement ${ }^{9}$. Cependant, l'impact réel de ces transferts sur le développement se révèle extrêmement délicat à quantifier. Si leur rôle dans l'allègement de la pauvreté au niveau local est aujourd'hui avéré par de nombreux travaux, par exemple sur les associations de migrants ouestafricains en France (Maliens, Sénégalais), l'ensemble des autres conséquences reste très difficilement mesurable ${ }^{10}$. Il faudrait se garder de justifier une diminution de l'aide vers certains pays en raison du volume des envois. Or, dans le cas de l'Afrique australe, on connaît aujourd'hui peu de choses sur le rôle des transferts dans le développement des pays d'origine.

6 Ce type d'étude établit aussi que les situations migratoires et la nature des économies en contact font grandement varier les conséquences à attendre de la migration ellemême et des mesures politiques qui peuvent être prises tant en matière de politique industrielle et commerciale qu'en matière de régulation de l'accès au territoire et au marché de l'emploi. Ainsi Schiff montre-t-il que l'ouverture des marchés au Nord et l'augmentation des investissements directs étrangers et de l'aide publique au développement en direction des pays en développement ont plus de chance de ralentir l'émigration depuis l'Europe de l'Est vers l'Union européenne que depuis l'Afrique vers l'Union européenne ou depuis l'Amérique latine vers les États-Unis ${ }^{11}$. La libéralisation du commerce entre l'Union européenne et les pays d'Afrique sub-saharienne pourrait même avoir pour conséquence une augmentation de la migration et une diminution du niveau de qualification des migrants ${ }^{12}$.

7 Ces éléments sont à remettre dans le contexte du continent africain où, si les migrations internationales ont régulièrement augmenté depuis trente ans avec un ralentissement ces dix dernières années, elles ont conservé de façon constante une part importante de réfugiés. En 1975, on estimait que l'Afrique comptait 9,9 millions de migrants internationaux dont 1 million de réfugiés, sur 82 millions de migrants dans le monde. En 2000, l'Afrique comptait 16,3 millions des 175 millions de migrants dans le monde. La même année, même si le nombre et la proportion des réfugiés avaient diminué par rapport à 1990, année record où les réfugiés représentaient $33 \%$ des migrants internationaux, ils demeuraient tout de même encore 3,6 millions, soit $22 \%$ de tous les migrants internationaux dans la région ${ }^{13}$.

8 L'Afrique australe a connu différentes phases dans une stratégie séculaire d'approvisionnement en main-d'œuvre de ses bassins miniers. La situation actuelle est à la fois le reflet d'une poursuite de ces dynamiques, au sein desquelles il ne faut pas négliger les stratégies d'exportation de main-d'œuvre développées par les états voisins, et une restructuration drastique du secteur minier et d'autres secteurs des économies des pays les plus privilégiés de la zone. Les flux anciens, à la fois transformés et maintenus dans la période post-apartheid, doivent être distingués de dynamiques totalement spécifiques à la situation actuelle. On peut citer entre autres facteurs essentiels: l'émergence d'un nouveau contexte politique favorisant la mise en place d'un régime de l'asile; la mise en place de nouveaux réseaux migratoires africains initiés par les classes moyennes dans les années 1980 en direction de la Namibie, du 
Botswana, puis des bantoustans et des centres urbains sud-africains, et poursuivis par de plus jeunes générations, moins qualifiées, fuyant l'instabilité politique de plusieurs régions du continent africain; et enfin, la réintégration de l'Afrique australe dans les réseaux migratoires mondiaux. Après avoir été tout au long de son histoire une région d'immigration, l'Afrique australe avait en effet connu une période de fermeture relative aux apports étrangers, en dehors d'une immigration "blanche» protestante encouragée notamment par les gouvernements sud-africain et namibien sous l'apartheid. Aujourd'hui, l'installation ou la réinstallation des diasporas mondiales chinoise, indienne, pakistanaise, israélienne et russe témoignent de cette inscription dans les phénomènes liés à la mondialisation.

9 Si la grande majorité des migrants internationaux en Afrique, comme ailleurs dans le monde, reste composée de migrants volontaires réguliers, la proportion des migrations forcées y demeure une constante à laquelle l'Afrique du Sud a dû s'adapter depuis sa réouverture au reste du monde à partir du début des années 1990. Cette composition particulière des migrations internationales africaines n'a pas forcément pour conséquence une moindre qualification de ce type de migrants, puisque plusieurs études montrent aujourd'hui que les populations de réfugiés en Afrique du Sud auraient un niveau de qualification supérieur à la moyenne de la population sudafricaine ${ }^{14}$. Il s'agit plutôt d'une forme de migration caractérisée par un statut administratif et socio-économique précaire, un vécu marqué par l'impossibilité de se projeter dans le futur et des représentations négatives de ce type de migrants au sein de la population autochtone. En outre, l'émergence d'un régime de l'asile en Afrique australe, à partir du début des années 1990, crée une possibilité supplémentaire de séjour légal pour différents migrants, qui autrement n'auraient pu s'installer dans ces pays. C'est probablement le cas de nombreux Congolais, Rwandais, Burundais et Somaliens qui, faute de ressources financières, ne peuvent prétendre à un séjour temporaire légal et pour lesquels l'asile représente un moyen de séjourner dans le pays. Cela a pour conséquence de brouiller les différentes catégories de permis de séjour puisque l'entrée temporaire pour tourisme ou pour études est utilisée pour pénétrer légalement sur le territoire avant de déposer une demande d'asile. Sans qu'il soit possible ici de développer l'ensemble des répercussions de l'émergence d'un régime de l'asile en Afrique australe, il faut noter que celui-ci a contribué à une réorganisation de la sous-région qui voit la création de zones d'attente dans le pourtour constitué par la Zambie, le Malawi, le Zimbabwe et le Mozambique.

10 L'Afrique du Sud et ses voisins botswanais et namibien partagent une autre spécificité paradoxale : la qualité de leurs systèmes éducatifs les exposent au phénomène de fuite de cerveaux tandis que l'incapacité de ces mêmes systèmes à se réformer devant le défi de la massification de l'éducation et de la transformation de leurs économies créent des déficits de compétences, particulièrement dans les secteurs potentiellement les plus dynamiques. En Afrique du Sud, la polémique est relativement récente et repose en partie sur l'idée d'un patriotisme moindre des populations blanches. Certaines déclarations du président Mandela sur la « fuite des Blancs » et sur l'émigration comme "révélateur des vrais Sud-africains » ont nourri cette perception. Pourtant l'Afrique du Sud a eu recours depuis la fin des années 1970 à des milliers d'Africains qualifiés pour combler le déficit de main-d'œuvre de ses bantoustans : l'étude de Kwesi Prah en 1989 affirme que ce "grand trek des talents" aurait compté environ 7000 Africains hautement qualifiés ${ }^{15}$. Dans les autres pays de la Southern African Development Community (SADC) comme la Namibie ou le Botswana, la «fuite des cerveaux » est 
beaucoup plus ancienne. Confrontés à la difficulté d'assurer le retour de leurs étudiants envoyés en Europe, ces pays ont investi sur la formation locale de leurs propres élites, non sans avoir recours aux autres "cerveaux" de la région qui occupent souvent aujourd'hui des positions élevées dans les universités et les administrations de ces pays.

11 Afrique du Sud, Botswana, Namibie sont donc au cœur des problématiques migratoires du continent: accusant les pays du Nord, comme le Royaume-Uni, le Canada ou l'Australie, de "piller» leurs ressources humaines les plus qualifiées, ils sont euxmêmes l'objet d'accusations similaires sur le continent africain. Depuis une dizaine d'années, les gouvernements de ces trois pays d'accueil affichent des politiques qui oscillent entre des mesures protectionnistes, la reconnaissance d'une situation de " vases communicants " (brain drain/brain gain) qui encourage plutôt des politiques de laisser-faire, et une réflexion sur la mise en place de politiques interventionnistes qui compenseraient et accompagneraient les effets les plus négatifs de ces récents bouleversements migratoires.

\section{L'Afrique australe post-apartheid : terminus et carrefour}

12 L'histoire des migrations en direction de l'Afrique australe a en effet subi ces quinze dernières années des mutations structurelles que l'on peut retracer en six points essentiels. Premièrement, la modernisation de l'économie sud-africaine a eu pour conséquence une quantité importante de licenciements qui se comptent aujourd'hui en centaines de milliers dans les secteurs minier, agricole, industriel. Alors qu'elle était retombée à moins de $40 \%$ en 1985 , la proportion de travailleurs étrangers dans le secteur minier oscille entre 50 et $60 \%$ depuis 1999, un taux similaire à celui des années 1970. En Afrique du Sud aujourd'hui, selon The Employment Bureau of South Africa (TEBA), environ $21 \%$ des mineurs seraient mozambicains (figures 1 et 2 ). Les mineurs étrangers licenciés se dirigent soit vers la migration irrégulière de survie, soit vers les sous-traitants qui trouvent là une main-d'œuvre peu chère et qualifiée. Ces mineurs, essentiellement basothos et mozambicains, constituent un bassin d'emploi tout à fait attractif pour les sous-traitants qui ont, de toute façon, de plus en plus souvent recours à la main-d'œuvre étrangère dans d'autres secteurs comme la foresterie et l'agriculture ${ }^{16}$. Malgré cette contraction du marché de l'emploi sud-africain - le taux de chômage officiel est d'environ $30 \%$, le taux officieux supérieur à $40 \%{ }^{17}$-, son attractivité ne semble pas avoir faibli pour les migrants «traditionnels» que sont les Basotho et les Mozambicains ${ }^{18}$, comme pour ceux venus du reste du continent ${ }^{19}$. 
Figure 1

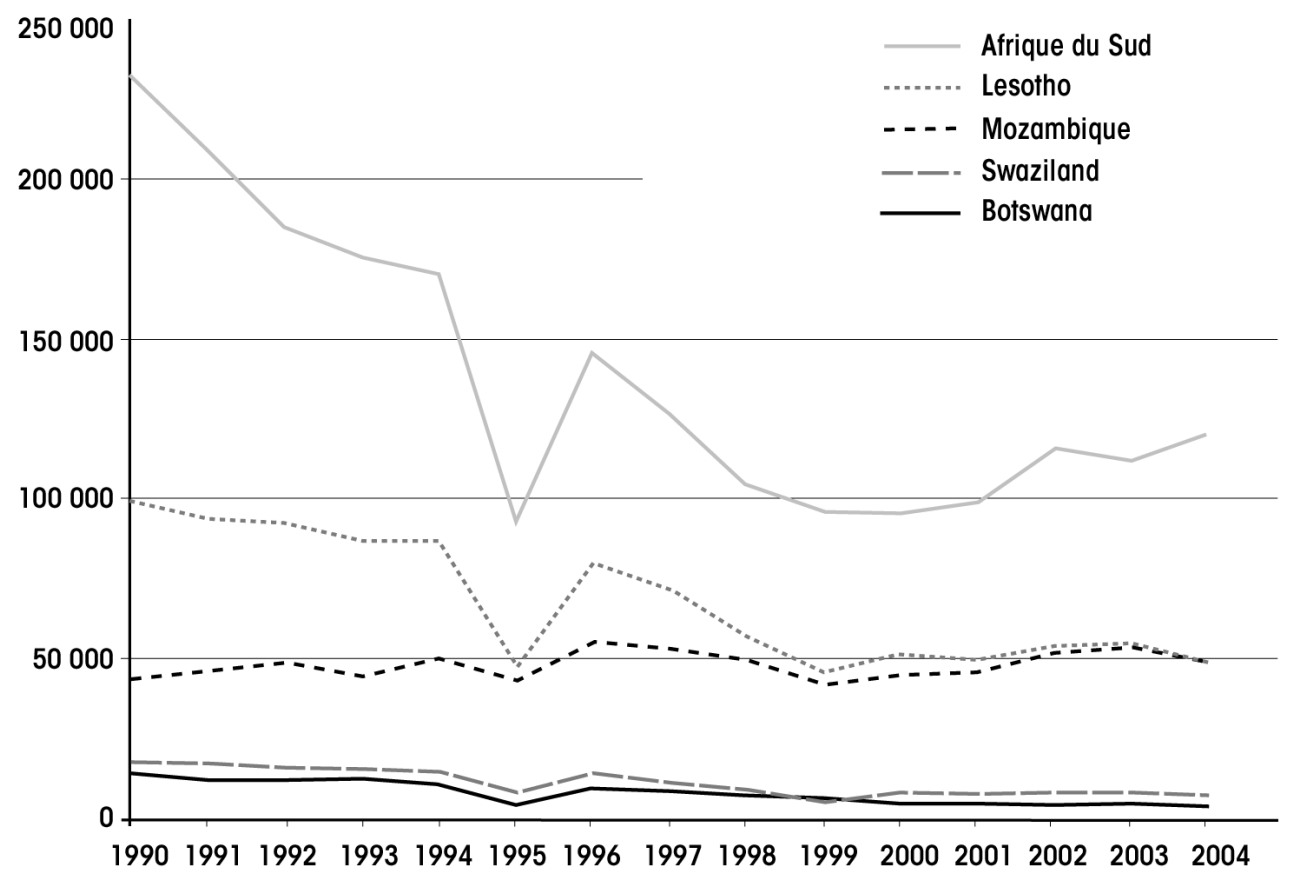

Nombre et origine des mineurs employés dans les mines sud-africaines

Figure 2

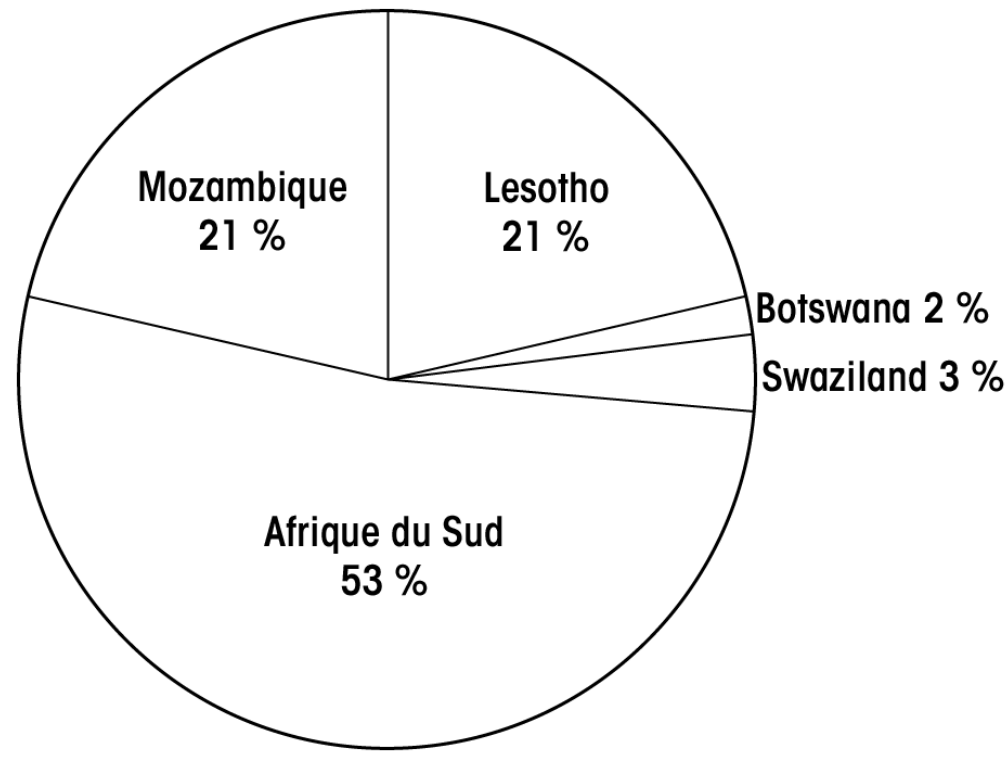

Répartition par pays d'origine des mineurs dans les mines suafricaines en 2004

Deuxièmement, les migrations vers l'Afrique australe présentent trois tendances démographiques spécifiques: la diversification des origines, le rajeunissement et la féminisation. Le grand changement de la période post-apartheid est l'arrivée de migrants venus d'Afrique et d'Asie. Après une domination continue des migrants venus 
d'Europe jusqu'en 1998, c'est d'Afrique que sont majoritairement originaires les immigrés permanents depuis 2000, l'Asie et l'Europe se partageant le reste des permis de résidence permanente presque à égalité (figure 3).

Figure 3

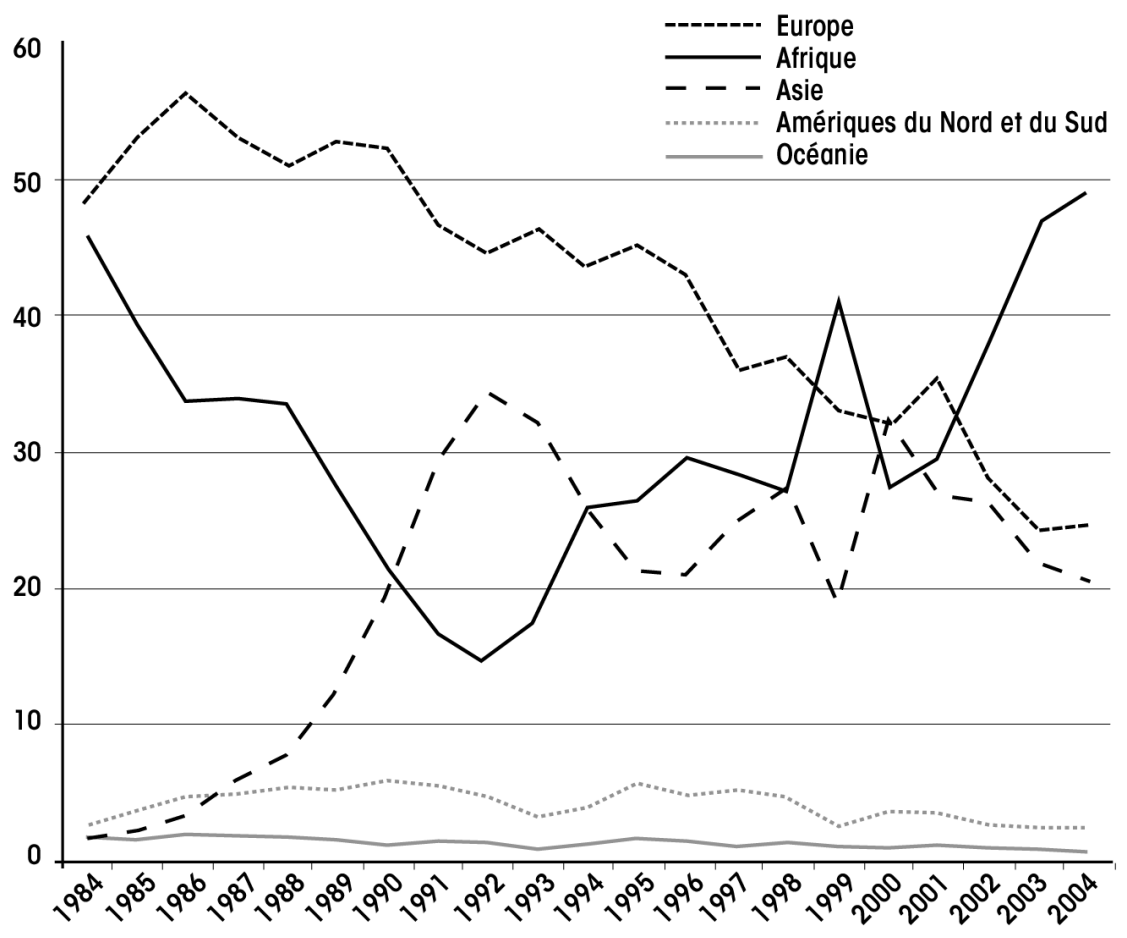

Composition de l'immigration permanente légale (en pourcentage) 1994-2004

Cela représente un changement majeur et sans précédent dans la composition de l'immigration permanente en Afrique du Sud depuis la création de l'Union : jamais les Africains n'avaient constitué la très large majorité des nouveaux résidents permanents. En valeur absolue, les chiffres restent modestes ( 5235 Africains sur 10714 résidences permanentes octroyées en 2004) et ne peuvent dans l'immédiat influer sur la composition démographique du pays. Mais cette tendance est à surveiller sur le long terme, d'autant plus que s'ajoutent à ces nouveaux résidents des migrants pratiquant des réseaux établis de longue date et des migrants temporaires usant de circuits plus récents. En effet, si la brèche juridique permettant l'immigration de non-Européens date de 1986, elle a surtout profité aux Africains qualifiés venus travailler dans les bantoustans sud-africains. Les travailleurs migrants des secteurs minier et agricole n'ont eu la possibilité d'accéder à l'immigration permanente qu'en 1996, sans d'ailleurs que cette opportunité remporte un franc succès. On est donc aujourd'hui en présence d'une augmentation non seulement de l'immigration permanente africaine, mais également de différentes formes de séjour qui, même s'ils sont officiellement ou officieusement temporaires (asile, permis de travail ou d'études, séjour irrégulier), s'inscriront de facto dans la durée en transformant la physionomie des sociétés de ces pays d'accueil. Historiquement, c'est la composition même des sociétés d'Afrique australe qui s'en trouve potentiellement modifiée à un rythme sans précédent. 

les hommes, particulièrement en ce qui concerne les migrations frontalières ${ }^{20}$, en partie en raison d'un phénomène de remplacement des hommes dans les secteurs les moins favorisés de l'économie sud-africaine comme l'agriculture. En outre, les femmes semblent également migrer aujourd'hui pour un ensemble d'autres raisons : commerce transfrontalier et international, affaires, études, mais aussi en tant que victimes de trafics d'êtres humains et prostituées. Enfin, ces nouvelles migrations se distinguent par leur rajeunissement, résultat de migrations de jeunes hommes célibataires essentiellement qui trouvent là un moyen d'échapper au poids socio-économique et parfois culturel des contraintes familiales ${ }^{21}$. Cette tendance s'explique aussi par l'attractivité des structures éducatives, particulièrement des enseignements secondaire et universitaire sud-africain, botswanais et namibien, qui reçoivent aujourd'hui une part croissante de l'élite intellectuelle africaine. Mais là aussi, l'Afrique du Sud est à la fois victime et bénéficiaire: une enquête récente a montré que les étudiants sudafricains envisagent essentiellement de poursuivre leur formation en Europe alors que les étudiants basothos, swazis, zimbabwéens et namibiens préfèrent demeurer en Afrique australe ${ }^{22}$. Les étudiants ressortissants de pays de la SADC représentent aujourd'hui $8 \%$ du total des inscriptions dans l'enseignement supérieur sud-africain ${ }^{23}$.

Troisièmement, la fin de l'apartheid a inséré l'Afrique du Sud dans un ensemble de réseaux commerciaux formels et informels à l'échelle régionale, continentale et internationale. Cette insertion repose en grande partie sur les réseaux migratoires transnationaux qui se sont structurés dans la période 1990-1995. Alors que différentes études sur le commerce urbain informel dans les années 1990 ne font aucune référence aux migrants ${ }^{24}$, ceux-ci prennent une visibilité évidente dès le milieu des années 1990 dans le commerce de rue, visibilité dont la réalité est confirmée par un nombre croissant d'analyses ${ }^{25}$.

Quatrièmement, la période post-apartheid a vu l'augmentation des migrations à caractère irrégulier, en particulier vers l'Afrique du Sud. Là non plus, le phénomène n'est pas nouveau dans une région où la réalité et les représentations liées à la notion d'«illegal alien» trouvent leurs racines en grande partie dans la législation sur les bantoustans et les lois de l'«influx control » régissant la mobilité des personnes sous l'apartheid. Mais l'ouverture des frontières, la normalisation des relations avec les états voisins et la relative prospérité des économies sud-africaine, botswanaise et namibienne ont suscité un phénomène d'appel d'air. Même si l'on peut raisonnablement penser que la majorité des migrants entrants demeurent réguliers ${ }^{26}$, le nombre d'irréguliers aurait augmenté sensiblement. Il n'est, par définition, pas aisé de fournir des chiffres précis sur leurs effectifs, au risque de retomber dans les polémiques qui ont émaillé ces calculs au sein des sciences sociales sud-africaines. Les estimations actuelles varient entre $500000^{27}$ et 4,1 millions ${ }^{28}$.

Cinquièmement, l'extrême pointe de l'Afrique australe est devenue une nouvelle destination pour les demandeurs d'asile et réfugiés du reste du continent. Longtemps pays émetteur de réfugiés sous l'apartheid, l'Afrique du Sud, d'abord avec la guerre civile mozambicaine, ensuite avec les nombreuses sources d'instabilité politique sur le continent dans la Corne puis la région des Grands Lacs, est devenu une terre d'asile. Ce sont près de 186000 demandeurs d'asile qui ont été officiellement enregistrés depuis la mise en place d'accords entre le gouvernement sud-africain et le Haut Commissariat aux réfugiés des Nations unies en 1993. Seuls 25800 ont aujourd'hui obtenu le statut de 
réfugié ce qui reflète un taux d'approbation de $13,87 \%$, taux relativement faible au niveau international ${ }^{29}$.

Enfin, la dernière caractéristique des migrations en direction de l'Afrique australe a trait à leur temporalité. Alors que la politique migratoire sud-africaine sous l'apartheid était caractérisée par la permanence de l'immigration "blanche » et l'impermanence de l'immigration "noire", la politique migratoire actuelle favorise l'impermanence, aussi bien à travers la législation existante que les représentations des migrants dans les discours officiels. Ainsi, l'ensemble de l'architecture sur laquelle repose cette politique est conçu pour limiter les installations durables et encourager le retour dans les pays d'origine. Le nombre de permis temporaires de travail s'est considérablement accru depuis la fin de l'apartheid, tout comme le nombre de visas touristiques, dont on sait qu'une partie dissimule en fait des entrées irrégulières. Ce chiffre est en effet passé de 3 millions en 1992 à 9,9 millions en $1999^{30}$. En revanche, l'immigration permanente légale, en dépit de sa diversification, a considérablement diminué. D'environ 14000 permis de résidence permanente en 1990, on est passé à 4000 à la fin des années 1990 pour revenir à environ 10000 par an en $2004^{31}$. Selon les chiffres officiels de l'immigration et de l'émigration légales, le solde migratoire est globalement négatif depuis 1995.

Des programmes de rapatriement volontaire des réfugiés mozambicains ont été appliqués par le Haut Commissariat aux réfugiés (HCR), et l'office international des migrations (OIM) en prévoit d'autres pour les réfugiés congolais et rwandais. En parallèle, trois campagnes de régularisation des migrants irréguliers ont eu lieu, mais sans que l'on puisse dire s'il s'agit là d'un succès considérable: en effet, alors que le gouvernement sud-africain avait avancé des chiffres allant jusqu'à 12 millions de demandes anticipées, le total des trois régularisations s'élève en tout à moins de 300000 personnes soit moins de $0,5 \%$ de la population sud-africaine ${ }^{32}$. Les reconduites à la frontière sont menées à grande échelle et avec une intensité qui ne faiblit pas depuis douze ans. Ce sont près de 1,6 million de personnes qui ont ainsi été reconduites à la frontière depuis $1993^{33}$. Cette politique touche plus particulièrement les ressortissants mozambicains, qui ont longtemps constitué près de $80 \%$ des reconduites à la frontière, y compris durant la période pendant laquelle ils auraient légitimement pu prétendre au statut de réfugiés.

21 Cette situation a constitué un cas ambigu de refoulement dissimulé, les accords internationaux passés avec le HCR ne permettant pas de protéger ces populations, victimes d'une politique sud-africaine d'immigration qui ne leur offrait aucun statut ${ }^{34}$. Mais cette politique de reconduite est également problématique par rapport aux implications socio-économiques qu'elle peut avoir sur ces populations mozambicaines et zimbabwéennes qui, depuis plus d'un siècle, ont eu recours à la migration vers l'Afrique du Sud et, plus récemment, vers le Botswana, comme stratégie de survie en période de crise. Le temps de séjour, raccourci en raison des arrestations et reconduites, et les gains économiques et matériels tirés du séjour sud-africain sembleraient être de plus en plus faibles, que le séjour soit légal, dans le cadre de contrats de sous-traitance, ou irrégulier. Cela pourrait avoir des répercussions à moyen terme sur l'équilibre de la sous-région.

Enfin, la xénophobie ambiante, confirmée par différents rapports ${ }^{35}$, n'encourage guère le désir d'installation permanente. La position des migrants interrogés dans différentes enquêtes, révèle une perception de la migration comme une ressource stratégique et 
temporaire et très peu de désirs d'installation durable à travers l'acquisition de la citoyenneté sud-africaine ${ }^{36}$. Il semble cependant qu'il soit trop tôt pour juger de ces phénomènes auprès de ces primo-arrivants. Les enquêtes menées auprès d'immigrés mozambicains sur des périodes plus longues par exemple -tendent à montrer leur degré d'intégration, y compris à travers leur acquisition parfois difficile de la nationalité sud-africaine ${ }^{37}$.

\section{Les défis politiques : migration et Renaissance} dans la nouvelle loi sud-africaine sur l'immigration (2002) et les différents décrets qui la régissent ${ }^{41}$ est handicapante pour les services du ministère de l'Intérieur sudafricain qui, depuis 2003, ne disposent donc plus de chiffres. À l'heure actuelle, seuls les entrées et départs des étrangers et des résidents permanents sont enregistrés aux frontières dans le système baptisé Movement Control System (MCS). Le ministère de l'Intérieur connaît cependant plusieurs mois de retard dans la saisie de ces données et les allées et venues des citoyens sud-africains ne sont pas enregistrées du tout.

Devant la pénurie de compétences, une ouverture politique à l'apport de main-d'œuvre qualifiée étrangère est notable depuis 1999. Le Livre blanc sur l'immigration (White Paper on International Migration) est explicite sur l'importance accordée par le 
gouvernement sud-africain à l'attraction de "personnes qualifiées en Afrique du Sud pour enrayer la fuite des cerveaux ${ }^{42}$ ". Cet objectif a été adopté officiellement par l' African National Congress (ANC) début 2001 et inscrit dans le préambule du dernier amendement en date de la loi sur l'immigration (octobre 2004) : il y est fait clairement mention d'une promotion de la croissance sud-africaine grâce à «l'emploi de maind'œuvre étrangère nécessaire " et à la facilitation de "l'entrée des personnes présentant des compétences ou des qualifications exceptionnelles ${ }^{43 "}$ ". Le Trésor sudafricain a ainsi annoncé son intention d'alléger la taxation des expatriés hautement qualifiés vivant en Afrique du Sud ${ }^{44}$.

Concrètement, le gouvernement sud-africain a choisi de mettre en œuvre une politique de quotas: les compétences recherchées sont ainsi déterminées annuellement par le ministre de l'Intérieur dans le cadre de la nouvelle loi de 2002. Différents dysfonctionnements ont émaillé la mise en place de ces quotas. Ceux-ci devaient préciser le type de compétences spécifiques ou de professions recherchées et être renouvelés annuellement. Or, depuis 2003, date d'entrée en vigueur du texte, les définitions de compétences sont demeurées vagues et n'ont jamais été renouvelées. En outre, l'écart entre le nombre de permis de travail accordés chaque année par le ministère de l'Intérieur sud-africain et les besoins réels tels qu'évalués par un rapport conjoint des ministères de l'Intérieur, du Travail et du Commerce et de l'Industrie en 2003 est énorme. L'Afrique du Sud délivre ainsi environ 10000 permis d'immigration permanente chaque année. Or le rapport interministériel de 2003 identifiait les besoins en termes de compétences à 740000 personnes.

L'Afrique du Sud a également fait le choix d'une politique d'incitation au retour des expatriés sud-africains. Trouvant ses racines dans un appel qu'aurait lancé Nelson Mandela à Trafalgar Square peu après sa libération, une campagne (dite Homecoming) a été menée pour inciter les expatriés sud-africains à rentrer au pays. Initiative conjointe du syndicat Solidarity et de la Company for Immigration ${ }^{45}$, cette opération aurait jusqu'ici aidé au retour de 500 personnes depuis son lancement en $2002{ }^{46}$, résultat insignifiant s'il en est.

30 Au total, les enquêtes les plus récentes insistent sur le fait qu'il est trompeur de parler de « fuite » des cerveaux. Une prise en considération de ces mouvements de personnes qualifiées révèle une circulation au niveau régional et peu de désir de nouveau départ après une première installation. Si l'attrait des pays du Nord est présent, l'intégration se produit plus significativement au niveau régional et à un rythme mesuré. Une enquête réalisée entre 1998 et 2001 par le Southern African Migration Project révélait ainsi que, sur l'échantillon d'étrangers qualifiés interrogés, $18 \%$ venaient de pays membres de la Southern African Development Community (SADC) et $41 \%$ étaient issus d'autres pays africains. Au Botswana, $77 \%$ de l'échantillon interrogé venait du continent africain dont une majorité de ressortissants de la SADC ${ }^{47}$. MacDonald et Crush en concluent que :

« Réfléchir au niveau régional conduit à remettre en question la notion de fuite des cerveaux. Si des Sud-Africains qualifiés travaillent au Lesotho, ou si des Zambiens qualifiés travaillent au Botswana, cela représente-t-il une perte de compétences pour leur pays d'origine ou une contribution à la construction et l'expansion du bassin de compétences de l'ensemble de la région ${ }^{48}$ ?"

31 Ce contexte régional offre cependant peu d'instruments de réflexion et de régulation en matière migratoire. Il est en effet frappant de constater que la SADC n'est pas parvenue à élaborer un outil de cette nature au niveau régional alors que le modèle 
Schengen, reposant sur l'abolition des frontières internes d'une zone d'intégration régionale, avait été un temps retenu par les États membres. Lancé en mars 1996, le Protocole sur la libre circulation des personnes dans la SADC a connu un destin tourmenté, révélateur des réticences sud-africaines, botswanaises et namibiennes face au défi migratoire de la région. D'inspiration libérale, ce protocole avait été conçu par son auteur, Charles Hove, comme le seul moyen de redresser les déséquilibres dus à un commerce asymétrique en faveur de l'Afrique du Sud en partant de l'idée d'une intégration de l'ensemble des facteurs de production, capital, main-d'œuvre et services. Ce protocole a pourtant rencontré l'opposition ferme de l'Afrique du Sud, du Botswana et de la Namibie, non convaincus par l'option libérale de substitution des moyens de production et inquiets des points suivants :

- la difficulté de garantir la sécurité des frontières extérieures ;

- une coordination hypothétique entre les pays étant donné le manque de ressources financières, techniques et humaines;

- les trop grandes disparités économiques entre les pays de la SADC risquant de mener à des flux incontrôlés vers les quelques pays les plus prospères, dont l'Afrique du Sud ;

- le risque de déstabilisation des conditions d'emploi de la main-d'œuvre dans les pays les plus prospères : chômage, pression à la baisse sur les salaires, perte d'influence des syndicats ;

- une augmentation de la xénophobie dans les pays d'accueil ;

- et un développement des réseaux criminels et des trafics divers.

En outre, le délai d'application prévu - dix ans - était considéré comme irréaliste.

Abandonné en 1997 en raison du rejet de l'Afrique du Sud, du Botswana et de la Namibie, ce projet a été relancé avec la rédaction d'un Protocole sur la facilitation des mouvements de personnes dans la SADC en 1998, sans plus de succès. Prévu sur vingt ans, ce nouveau protocole reprenait les mêmes objectifs que le précédent : abandon des visas dans la zone dans une première phase, liberté de résidence pour des périodes de trois ans renouvelables dans une deuxième phase, et liberté d'installation permanente dans une dernière phase. Mais, comme aucune clause n'obligeait les États membres à soumettre leur législation nationale à celle du Protocole, celui-ci était d'avance voué à l'échec. À l'occasion du sommet de la SADC à Grand Baie (île Maurice), en septembre 1998, et alors qu'il était prévu que ce Protocole sur la facilitation des mouvements de personnes soit adopté, un énième report de ratification était annoncé, fixant de nouveaux objectifs pour 1999, là encore non respectés. De manière caractéristique, la déclaration annonçant ce nouveau report précisait que, dans l'attente de la ratification finale: «[...] les Ètats membres devront faciliter les entrées sur leur territoire des ressortissants de la SADC sur la base d'accords bilatéraux et dans leur législation nationale ${ }^{49}$."

Le débat sur la libre circulation de la main-d'œuvre dans la région fut ainsi enterré pendant quelque temps. Mais le gouvernement sud-africain devait le relancer en 2001 lorsque le porte-parole de la présidence sud-africaine, Essop Pahad, déclara dans un discours au Parlement, en pleine discussion houleuse du nouveau projet de loi sur l'immigration:

« Nous ne pouvons pas, en tant que Sud-Africains, échapper au fait que, tôt ou tard, nous devrons reconnaître que nous ne pouvons disposer d'une zone de libreéchange sans une liberté de circulation de la main-d'œuvre dans cette région. [...] Cela nous permettrait de répondre aux difficultés que rencontrent les populations de notre région d'une manière différente, et non plus seulement en fonction des mouvements de capitaux et des échanges commerciaux. Nous parlons de la réalité 
des flux de population, de la libéralisation des flux de main-d'œuvre et de la liberté

de circulation dans notre région ${ }^{50}$. » marquent leur préférence pour deux types d'instruments de régulation des migrations : les protocoles sectoriels comme le Protocole sur l'éducation et la formation pour encourager la mobilité des étudiants et des chercheurs, et les accords bilatéraux de coopération, entre l'Afrique du Sud et le Royaume-Uni notamment, afin de réguler l'émigration de personnels qualifiés. L'Union africaine, à travers son Cadre stratégique pour une politique migratoire (Strategic Framework for a Policy on Migration) publié en mars 2004, n'offre que quelques points de repère sans véritables moyens ni instruments coercitifs. Enfin, le NEPAD insiste particulièrement sur la mise en place d'une base de données sur la diaspora africaine dans le monde. L'objectif est d'investir sur les ressources humaines africaines en renversant le phénomène de fuite des cerveaux et en maîtrisant le déploiement de la diaspora. Les paragraphes 124 et 125 du document du NEPAD font référence à ces objectifs :

- renverser le brain drain et le transformer en brain gain pour l'Afrique ;

- construire et retenir à l'intérieur du continent le capital humain critique pour le développement africain ;

- développer des stratégies pour l'utilisation du savoir-faire et des compétences scientifiques et technologiques des Africains de la diaspora pour le développement de l'Afrique.

\section{Cosmopolitisme et construction nationale}

Le défi migratoire de l'Afrique australe va au-delà d'une seule gestion " rationnelle » des flux de compétences. Premièrement, parce qu'une partie non négligeable de ces flux est le fruit de migrations séculaires transfrontalières de personnes peu ou pas qualifiées. Il y a donc des enjeux liés aux équilibres socio-économiques fragiles que des politiques migratoires ultra-sélectives ne peuvent prendre suffisamment en compte. La mesure adoptée entre l'Afrique du Sud et le Mozambique depuis septembre 2004, qui abolit les frais de visa pour les séjours inférieurs à 30 jours, permet ainsi à de 
nombreuses populations frontalières de migrer légalement. Deuxièmement, parce que ces flux sont également le résultat de déséquilibres politiques importants et ne sont pas nécessairement réversibles même en cas de résolution du conflit. Le cas des réfugiés mozambicains ou rwandais en est une bonne illustration. Sans compter que d'autres conflits ou situations d'instabilité politique perdurent dans la région à plus ou moins grande intensité (région orientale de la République démocratique du Congo, Zimbabwe, Somalie, Soudan). L'Afrique australe est devenue une destination d'élection: elle a acquis le statut de recours en cas de crise pour nombre de populations d'Afrique centrale et même d'Afrique de l'Ouest. La diffusion de la culture de consommation sudafricaine par le biais de ses expatriés, de ses commerces de grande distribution, de ses compagnies de téléphonie mobile, de sa musique et de ses chaînes de télévision par satellite, contribue à la construction de ces représentations. Il est frappant de constater la rapidité d'émergence de ces images qui, en une dizaine d'années, sont devenues prégnantes dans les imaginaires des Africains du bassin du Congo. Les plus anciennes générations de mineurs qualifiés et d'ingénieurs du Katanga congolais avaient certes quelques souvenirs de campagnes de recrutement dans les mines sud-africaines, mais pour la majeure partie des Africains d'Afrique centrale, l'Afrique du Sud constituait, il y a encore peu, le "pays de l'apartheid », c'est-à-dire une impasse qui ne figurait pas dans les schémas migratoires.

Aujourd'hui, l'Afrique du Sud et le Botswana sont aussi conçus comme des destinations de transit, des tremplins vers d'autres cieux (Europe, Amérique du Nord). Ainsi, les Congolais et les Somalis en particulier utilisent l'Afrique du Sud pour émigrer vers la Grande-Bretagne, les États-Unis et le Canada. Différents facteurs contribuent à cela. Les possibilités économiques permettent à ces migrants de se constituer une épargne afin de financer leur passage. Des relais à la fraude documentaire et aux réseaux de migration irrégulière au sein des administrations du ministère de l'Intérieur, de la police et de la sécurité des aéroports rendent le passage possible. Enfin, les sociétés au Nord (Royaume-Uni, Canada, États-Unis) attirent les réfugiés qui espèrent y obtenir, à plus ou moins long terme, un statut socio-économique leur permettant d'entre-prendre des études, de travailler et de procéder au rapprochement familial. Ce phénomène de tremplin contribue à faire vivre la migration comme un passage ou une condition temporaire. Il a également des répercussions dans l'organisation politique de ces migrants et, notamment, dans leur relation à la ville comme espace de socialisation, voire pour certains, de première socialisation aux formes les plus achevées de l'État. Les plus jeunes primo-arrivants somaliens ou congolais, en particulier, ont été familiarisés avec des formes de pouvoir assez éloignées de la réalité sud-africaine, ou du moins, qui correspondent à une réalité de l'État - la corruption par exemple-, que les gouvernements sud-africain, namibien ou botswanais considèrent contraire à leurs entreprises respectives de construction nationale.

On s'aperçoit aujourd'hui de la diversité des situations migratoires en Afrique australe, mais le contexte de pénurie d'études ethnographiques ne permet pas (encore) de connaissance fine de ces situations, des circuits utilisés, ni des réseaux dans leurs ramifications, leur mode opératoire, leur étendue, leur fonctionnalité. Si, pour certains migrants qualifiés, l'Afrique australe est une bonne alternative à l'Europe, pour d'autres, elle n'est qu'une étape sur une route beaucoup plus longue, durant laquelle les liens avec le pays d'origine et les nouvelles appartenances entretiennent des relations complexes. C'est en partie le cas de populations moins qualifiées prêtes à « tenter leur 
chance ", dans un état d'esprit assez similaire aux mentalités d'aven-turier décrites en Afrique de l'Ouest autour des pôles de Nouadhibou en Mauritanie et Ceuta et Melilla au Maroc ${ }^{52}$. Plus simplement, il semble qu'on observe une intensification des migrations transfrontalières de proximité, à la frontière angolo-namibienne ${ }^{53}$ et aux frontières zimbabwéenne et mozambicaine avec l'Afrique du Sud.

Cette diversité ne doit pas dissimuler ce que L. Landau désigne comme « l'affrontement des allochtones postmodernes et des autochtones modernes ${ }^{54}$ ». Par cette image, Landau souligne le défi posé par la présence des migrants qui s'efforcent d'échapper à l'emprise étatique. Johannesburg, comme d'autres grandes métropoles ou capitales africaines, accueille un nombre croissant de ces migrants en perpétuel transit ${ }^{55}$. À travers leur présence, c'est une part non négligeable de l'économie urbaine qui échappe à l'emprise de l'État, que ce soit par le biais du commerce informel, des trafics en tout genre, des envois informels de fonds, de la fraude documentaire ou fiscale, ou simplement par des stratégies qui permettent à des individus de se dérober à toute existence légale. Ainsi, moins de $20 \%$ des enfants d'étrangers à Johannesburg seraient inscrits à l'état civil, et le Haut Commissariat aux réfugiés considérerait que moins de $20 \%$ des demandes d'asile en Afrique du Sud sont justifiées ${ }^{56}$. Face à cela, l'avènement de démocraties représentatives en Afrique du Sud ou en Namibie a permis depuis peu aux majorités autochtones de reconquérir, en le surinvestissant, le terrain à la fois symbolique, politique et spatial, des villes et des municipalités. Ces majorités perçoivent la présence insaisissable d'étrangers comme un obstacle à l'entreprise de modernisation que représente la construction nationale post-apartheid. Au-delà du discours classique accusant l'étranger le plus démuni de profiter des maigres ressources socio-économiques de l'État ${ }^{57}$, l'Afrique du Sud voit se développer des discours associant autochtonie et modernisation d'une part, et allochtonie et archaïsme de l'autre. Dans l'Afrique du Sud post-apartheid, l'étranger (africain en particulier), par ses stratégies (réelles ou fantasmées) d'évitement de l'État, constituerait une entrave à l'entreprise de modernisation nationale, fondée sur un modèle politique transitionnel et un constitutionnalisme novateur, mais également sur la réalisation d'une unité trans-ethnique et l'investissement dans les nouvelles technologies. Il peut paraître certes exagéré de considérer Johannesburg aujourd'hui comme une "nowhereville» (ville de nulle part) qui serait située en Afrique du Sud sans appartenir à l'Afrique du $\mathrm{Sud}^{58}$ : ce serait là conférer un poids trop grand à la présence étrangère d'une part, et trop faible à l'emprise de l'État de l'autre. Mais il est crucial de retenir cette perspective potentiellement irréductible de la relation allochtone-autochtone dans l'Afrique australe contemporaine, partagée entre un cosmopolitisme revendiqué plutôt par les élites et la montée des nationalismes post-apartheid. Sans compter que face à cela, une partie des migrants entretient cet état intermédiaire dans lequel, contrairement aux nationaux, la citoyenneté et ses corollaires ne sont pas des atouts mais plutôt des limites posées à une mobilité sur laquelle repose encore en grande partie les stratégies de survie au niveau régional. Il est encore difficile d'affirmer quelles seront les configurations sociales qui se dégageront de ces visions apparemment divergentes de l'appartenance, mais il faudra sans conteste prendre en compte cette dynamique pour comprendre à la fois la construction de l'État post-apartheid en Afrique du Sud, en Namibie et au Botswana, et le devenir de l'Afrique australe tout entière. 


\section{BIBLIOGRAPHIE}

- AMISI B. et R. BALLARD, «In the Absence of Citizenship : Congolese Refugee and Orga-nisation in South Africa ", Forced Migration Working Paper Series, Forced Migration Studies Programme, Johannesburg, $n^{\circ}$ 16, avril 2005.

- BANQUE MONDIALE, «La migration peut améliorer le bien-être et réduire la pauvreté », selon Perspectives économiques mondiales 2006, communiqué de presse, synthèse du rapport de la Banque mondiale « The Economic Implications of Remittances and Migration (Implications économiques des envois de fonds et de la migration) », octobre 2005, http://web.worldbank.org, accédé le 28 décembre 2005.

- BEINE M., F. DOCQUIER et H. RAPOPORT, « Brain Drain and LDCs' Growth : Winners and Losers » (July 2003), Institut zur Zukunft der Arbeit (IZA) Discussion Paper No 819. Disponible sur SSRN : http:// ssrn. com/abstract=434542. Consulté le 27 mars 2006.

- BLANKLEY W., « Brain Circulation - A Work-able Answer to the Brain Drain ? ", Rapport présenté lors d'une consultation publique du Conseil de l'immigration sud-africain, HSRC, Pretoria, 2003.

- BHABHA H. (éd.), Nation and Narration, Londres et New York, Routledge, 1990.

- BLION R., «Économies et transferts migratoires, révélateurs des contradictions entre politiques et pratiques migratoires ", in R. BLION et I. RIGONI, D'un voyage à l'autre. Des voix de l'immigration pour un développement pluriel, Paris, Institut Panos/Karthala, 2001.

- Bouillon A. (dir.), Immigration africaine en Afrique du Sud. Les migrants francophones des années 1990, Paris, Institut français d'Afrique du Sud/Karthala, 1999.

- BROOKES M. et T. HINKS, « Le fossé “racial”. L'accès à l'emploi dans la "nouvelle” Afrique du Sud », in P. Guillaume, N. PÉJOUT et A. WA KABWE-SEGATTI, L’Afrique du Sud dix ans après, Transition accomplie ?, Paris, Institut français d'Afrique du Sud/Karthala, 2004, pp. 53-78.

- CASTELls M. et A. PORTES, « The World Underneath : The Origins, Dynamics, and Effects of the Informal Economy », in A. PORTES, M. CASTELLS et L. BENTON (éds), The Informal Economy Studies in Advanced and Less Developed Countries, Baltimore, Johns Hopkins University Press, 1989, pp. 11-37.

- CASTELls M., The Rise of the Network Society, Oxford, Basil Blackwell, 1996.

- COHEN R., « Transnational Social Move-ments : an Assessment ", Economic and Social Research Council (ESRC) Trans-national Communities Programme, Working Paper No 10, Oxford, 1998.

- COMMISSION MONDIALE SUR LES MIGRATIONS INTERNATIONALES, Rapport « Les migrations dans un monde interconnecté : nouvelles perspectives d'action », New York, Global Commission on International Migration (GCIM), 2005.

- CONSULTATIVE GROUP TO ASSIST THE POOR, Supporting remittances in Southern Africa. Estimating Market Potential and Assessing Regulatory Obstacles, 16 May 2005, http:// www.cgap.org/

SAfricaRemittances/3.html, consulté le 29 mai 2005.

- Coplan D., In the time of Cannibals : The Word Music of South Africa's Basotho Migrants, Chicago, University of Chicago Press, 1994

- Coplan D., « Damned If We Know : Public Policy and the Future of the Migrant Labour System », in Labour Migrancy in Southern Africa : Prospects for Post-Apartheid Transformation, Southern African Labour Monographs 3/95, Labour Law Unit, University of Cape Town, 1995 
- COVANE L., Migrant Labour and Agriculture in Southern Mozambique with Special Reference to the Lower Limpopo Valley 1920-1992, Londres, Institute of Commonwealth Studies (ICS), 1996.

- COVANE L. et J. MACARINGUE, Preliminary Findings of a Survey on Undocumented Migration from Mozambique to South Africa, Maputo, Arquivo do Patrimonio Cultural (ARPAC), non publiée, 1998.

- CROSS C., « Migrant Workers' Remittances and Micro-Finance in South Africa », Report, Social Finance Programme, Pretoria, Human Sciences Research Council, 2003.

- CRUSH J., « The Discourse and Dimensions of Irregularity in Post-Apartheid South Africa », International Migration, New York, Centre for Migration Studies, vol. 37, n 1, 1999.

- CRUSH J., « Northern Gateway : Cross-Border Migration between Namibia and Angola », Migration Series, Queens Uni-versity, Kingston, Southern African Migra-tion Project, n 38, 2005.

- CRUSH J., A. JEEVES et D. YUdELMAN, South Africa's Labor Empire : A History of Black Migrancy to the Gold Mines, Boulder et Le Cap, Westview Press et David Philip, 1991

- CRUSH J. et D. MCDONALD, « Transnationalism, African Immigration, and New Migrant Spaces in South Africa : an Intro-duction ", Canadian Journal of African Studies, vol. 34, n 1, 2000, pp. 1-19.

- DEPARTMENT OF HOME AFFAIRS, Annual Reports, 1990-2004, Government of the Republic of South Africa.

- DoDSON B., « Women on the Move », Migration Series, Queens University, Kingston, Southern African Migration Project, $\mathrm{n}^{\circ}$ 9, 1998.

• ENSOR L., « Relief in the Cards for Skilled Expats », Business Day, 24 février 2005.

- GLICK SCHILLER N., L. BASCH et C. BLANC-SZANTON (éds), Towards a Transnational Perspective on Migration : Race, Class, Ethnicity and Nationalism Reconsidered, New York, New York Academy of Sciences, 1992.

- GoloobA-MUTEBI F., « Witchcraft, Trust and Reciprocity Among Mozambican Refugees and their South African Hosts in a Lowveld Village », Forced Migration Working Paper Series, Johannesburg, Forced Migration Studies Programme, ${ }^{\circ}$ 9, juin 2004.

- HANDMAKER J., N. JOHNSTON et J. SCHNEIDER, The Status « Regularisation » Programme for Former Mozambican Refugees in South Africa, Johannesburg, Lawyers for Human Rights/ University of the Witwatersrand Research Programme, 2001.

- HANNERZ U., Transcontinental Connections : Culture, People, Places, Londres, Routledge, 1996.

- HERBST J., States and Power in Africa. Comparative Lessons in Authority and Control, Princeton, Princeton University Press, 2000.

- HUMAN RIGHTS WATCH, "Prohibited Persons" Abuse of Undocumented Migrants, Asylum Seekers and Refugees in South Africa, New York, Human Rights Watch, 1998.

- HUMAN SCIENCES RESEARCH COUNCIL, « Flight of the Flamingos », rapport pour le Conseil national de l'innovation sud-africain, Pretoria, HSRC Press, 2004.

-JeEVEs A., Migrant Labour in South Africa's Mining Economy, Kingston et Montreal, McGill-Queen's Press, 1985.

- KIHATO C. et L. LANDAU, «The Uncaptured Urbanite : Migration and State Power in Johannesburg ", Forced Migration Working Paper Series, Johannesburg, Forced Migration Studies Programme, $\mathrm{n}^{\circ} 25$, mars 2006. 
- LANDAU L., « Transplants and Transients : Nativism, Nationalism and Migration in Inner-City Johannesburg ", Forced Migration Working Paper Series, Forced Migration Studies Programme, Johannesburg, $\mathrm{n}^{\circ}$ 19, juillet 2005.

- LUBKEMANN S., « The Transformation of Transnationality among Mozambican Migrants in South Africa », Canadian Journal of African Studies, vol. 34, n 1, 2000, pp. 41-63.

- MCDONALD D. (dir.), On Borders : Perspectives on International Migration in Southern Africa, New York, Southern African Migration Project, StMartin's Press, 2000.

- MACDONALd D. et J. CRUSH (éds), Destinations Unknown. Perspectives on the Brain Drain in Southern Africa, Pretoria, Africa Institute of South Africa, Southern African Migration Project, 2002.

- MCDONALD D., L. MASHIKE et C. GOLDEN, « The Lives and Times of African Migrants and Immigrants in Post-Apartheid South Africa ", Migration Series, Queens University, Kingston, Southern African Migration Project, $\mathrm{n}^{\circ}$ 13, 1999.

- MCDONAld D., L. zinYAMA, J. GAY, F. DE VletTer et R. MATTES, « Challenging Xenophobia : Myths and Realities About Cross-Border Migration in Southern Africa », Migration Series, Queens University, Kingston, Southern African Migration Project, n 7, 1998.

- MORRIS A., Bleakness and Light : Inner-City Transition in Hillbrow, Johannesburg, Wits University Press, 1999.

- MUNDELL R., «International Trade and Factor Mobility », American Economic Review, XCVII, juin 1957.

- NEVES J., O trabalho Migratório de Moçambique para a Rodésia do Sul, 1913-1958/60, Maputo, Universidade Pedagógica, 1990.

- office des migrations internationales, Rapport Mondial sur les Migrations, 2005.

- Ouchо J. O., Regional Integration and Intra- and Inter-Sub Regional Labour Mobility in Eastern and Southern Africa, Gaborone, University of Botswana, mimeo, 1997.

- PATON B., Labour Export Policy in Southern Africa, Harare, University of Zimbabwe Press, 1995.

- PEBERDY S. et C. ROGERSON, « Enclave Entrepreneurs ? Non-South African Entrepreneurs in South Africa's Informal Sector and Small and Medium Enterprises », Institute of Migration and Ethnic Studies, University of Amsterdam, Netherlands, 1999.

- PORTES A., « Globalization from Below : The Rise of Transnational Communities », in W. SMITH et R. KORCZENWICZ (dir.), Latin America in the World Economy, Westport, Greenwood Press, 1996.

- POLZER T., «Adapting to Changing Legal Frameworks : Mozambicans Refugees in South Africa », Forced Migration Working Paper Series, Forced Migration Studies Programme, Johannesburg, $\mathrm{n}^{\circ} 17$, mai 2005.

- POUTIGNAT P. et J. STREIFF-FÉNART, « De l'aventurier au commerçant transnational : trajectoires de migrants sub-sahariens en transit à Nouadhibou (Mauritanie) », 2005, communication au colloque du Cédéj, Circulations migratoires et reconfigurations territoriales entre l'Afrique noire et l'Afrique du Nord, 17-18 novembre 2005, Le Caire.

- PRAH K., «The Bantustan Brain Gain : A Study into the Nature and Causes of Brain Drain from Independent Africa to the South African Bantustans », Southern African Studies Series No 5, Roma, National University of Lesotho, 1989. 
- PRESTON-WHITE E. et C. ROGERSON (dir.), South Africa's Informal Economy, Le Cap, Oxford University Press, 1990.

- REITZES M., « Counting the Uncountable ? Undocumented Migrants in South Africa », Pretoria, Department of Labour, 1998.

- REITZES M. et S. BAM, « Citizenship, Immigration, and Identity in Winterveld, South Africa », Canadian Journal of African Studies, vol. 34, $\mathrm{n}^{\circ}$ 1, 2000, pp. 80-100.

- REPUBLIC OF SOUTH AFRICA, White Paper on International Migration, Pretoria, Govern-ment Printers, 1999.

- REPUBLIC OF SOUTH AFRICA, Immigration Act, 2002 (Act. No 13 of 2002).

- REPUBLIC OF SOUTH AFRICA, Immigration Amendment Act , 2004 (Act No 19 of 2004).

- ROGERSON C., «African Immigrant Entrepreneurs and Johannesburg's Changing Inner City », Africa Insight, Pretoria, Africa Institute of South Africa, vol. 27, n 4, 1997.

- schiff M., « South-North Migration and Trade. A Survey », Policy Research Working Papers, n 1696, Washington, The World Bank International Economics Department, International Trade Division, 1996.

- SCHAPERA I., Migrant Life and Tribal Life : A Study of Conditions in the Bechuanaland Protectorate, Le Cap, Oxford University Press, 1947.

- SOUTH AFRICAN HUMAN RIGHTS COMMISSION, Report into the Arrest and Detention of Suspected Undocumented Migrants, Parktown, Johannesburg, 19 mars 1999.

- SOUTHERn AfRicAn MigRATION PRojeCt, Potential Skills Base Survey, 2003, disponible à l'adresse http:// www.queensu.ca/samp/ mig_db/mig_db.php, consulté le 23-03-2006.

- STREEK B., «Significant Steps towards Free Movement of Labour in SADC Region », The Mail \& Guardian, 9 et 15 novembre 2001.

- VAN DER WeSTHUizen L., Migration of Skills in South Africa, Patterns, Trends and Challenges, Immigration Advisory Board, 2005 Conférence des Nations unies sur le commerce et le développement, Country Fact Sheet 2004, South Africa.

- VAN ONSELEN C., Chibaro : African Mine Labour in Southern Rhodesia, 1900-1933, Londres, Pluto Press, 1976.

-WA KABWE-SEGATTI A., « Du rapatriement volontaire au refoulement dissimulé : les réfugiés mozambicains en Afrique du Sud », Politique Africaine, $n^{\circ}$ 85, mars 2002.

- «Immigration Numbers a Misperception », UN Integrated Regional Information Net-works (IRIN), 13 octobre 2005.

\section{NOTES}

1. Nous renvoyons à la bibliographie aux auteurs suivants : pour le Botswana: I. Schapera et J. O. Oucho. Pour l'Afrique du Sud : C. van Onselen; A. Jeeves ; J. Crush, A. Jeeves et D. Yudelman ; D. Coplan 1994 et 1995 ; B. Paton; C. Rogerson. Pour le Mozambique : L. Covane ; L. Covane et J. Macaringue ; J. Neves.

2. L'Afrique australe offre des ressources statistiques relativement denses par rapport au reste $\mathrm{du}$ continent. Les principales données sont disponibles à travers l'institut sud-africain de la statistique Statistics South Africa, qui fournit les chiffres des recensements nationaux de 1996 et 
2001, les chiffres des ministères de l'Intérieur sud-africain et botswanais, les chiffres du Haut Commissariat aux réfugiés et les chiffres des compagnies minières. Des problèmes spécifiques de mauvaise gestion des données (perte), l'absence d'informatisation de certains postes frontaliers et l'absence de centralisation des informations concernant les réfugiés constituent une limite à la fiabilité des statistiques sud--africaines notamment. En outre, comme dans les pays industrialisés, l'évaluation du nombre des migrants irréguliers et la catégorisation des personnes entrant sur le territoire sont sujets de polémiques récurrentes ; il faut donc considérer davantage ces chiffres comme des indicateurs que comme des données absolues.

3. Enquêtes du programme Southern African Migration Programme (http://www.queensu.ca/samp) et du Forced Migration Studies Programme de l'université de Witwatersrand (http://www.wits.ac.za/ fmsp).

4. L'origine et le volume exacts de ces investissements directs étrangers sont encore mal connus, mais l'institut Edge aurait relevé 921 investissements sur le continent africain réalisés par 287 compagnies sud-africaines depuis 1994 et certaines sources donneraient l'Afrique du Sud comme premier investisseur africain. Elle l'est déjà en Zambie, au Mozambique, au Zimbabwe, au Malawi, en Namibie et au Botswana. Entretien avec Stephen Gelb, Edge Institute, 28 mars 2006. Voir également Conférence des Nations unies sur le commerce et le développement, Country Fact Sheet 2004, South Africa.

5. En référence à l'article de A. Portes, «Globalization from Below: The Rise of Transnational Communities ", in W. Smith et R. Korczenwicz (dir.), Latin America in the World Economy, Westport, Greenwood Press, 1996.

6. Banque mondiale, «La migration peut améliorer le bien-être et réduire la pauvreté ", selon Perspectives économiques mondiales 2006, communiqué de presse, synthèse du rapport de la Banque mondiale « The Economic Implications of Remittances and Migration (Implications économiques des envois de fonds et de la migration) ", octobre 2005, http://web.worldbank.org, accédé le 28 décembre 2005 ; Commission mondiale sur les migrations internationales, Rapport «Les migrations dans un monde interconnecté : nouvelles perspectives d'action ", New York, Global Commission on Inter-national Migration (GCIM), 2005.

7. M. Schiff, "South-North Migration and Trade. A Survey", Policy Research Working Papers, $\mathrm{n}^{\circ}$ 1696, Washington, The World Bank International Economics Department, International Trade Division, 1996.

8. R. Mundell, «International Trade and Factor Mobility ", American Economic Review, XCVII, juin 1957, pp. 321-335.

9. Les envois de fonds comptabilisés vers les pays en voie de développement se sont élevés à 170 milliards de dollars américains en 2005, contre environ 175 milliards d'investissements directs étrangers et 75 milliards d'aide publique au développement. Banque mondiale, rapport cit., 2005.

10. R. Blion, «Économies et transferts migratoires, révélateurs des contradictions entre politiques et pratiques migratoires", in R. Blion et I. Rigoni, D'un voyage à l'autre, Des voix de l'immigration pour un développement pluriel, Chapitre 3 : «Les revenus de la migration, une chance pour le développement? ", Paris, Institut Panos/Karthala, 2001.

11. M. Schiff, op.cit., p. 20.

12. Ibid.

13. Office des migrations internationales, Rapport mondial sur les migrations, 2005.

14. Voir les travaux du Southern African Migration Project (Potential Skills Base Survey, 2003, disponible à l'adresse http://www.queensu.ca/samp/mig_db/mig_db.php, consulté le 23 mars 2006) et du Forced Migration Studies Programme (http://www.wits.ac.za/fmsp).

15. K.Prah, «The Bantustan Brain Gain : A Study into the Nature and Causes of Brain Drain from Independent Africa to the South African Bantustans », Southern African Studies Series No 5, Roma, National University of Lesotho, 1989. 
16. J. Crush et al., «Undermining Labour: Migrancy and Sub-contracting in the South Africa Gold Mining Industry », Migration Series, Queens University, Kingston, Southern African Migration Project, $\mathrm{n}^{\circ} 15,1999$.

17. Pour une compréhension de la situation de l'emploi en Afrique du Sud, voir M. Brookes et T. Hinks, «Le fossé "racial". L'accès à l'emploi dans la "nouvelle" Afrique du Sud", in P. Guillaume, N. Péjout et A. Wa Kabwe-Segatti, L'Afrique du Sud dix ans après. Transition accomplie?, Paris, Institut français d'Afrique du Sud/Karthala, 2004, pp. 53-78.

18. L. Covane et J. Macaringue, op. cit.

19. A. Bouillon (dir.), Immigration africaine en Afrique $d u$ Sud. Les migrants francophones des années 1990, Paris, Institut français d'Afrique du Sud/Karthala, 1999; A. Morris, Bleakness and Light: Inner-City Transition in Hillbrow, Johannesburg, Wits University Press, 1999. B. Maharaj et V. Moodley, « New African Immigration to the Durban Region », Canadian Journal of African Studies, vol. 34, $\mathrm{n}^{\circ} 1,2000$, p.149-160.

20. B. Dodson, «Women on the Move », Migration Series, Queens University, Kingston, Southern African Migration Project, $\mathrm{n}^{\circ}$ 9, 1998.

21. C. Kihato et L. Landau, "The Uncaptured Urbanite: Migration and State Power in Johannesburg», Forced Migration Working Paper Series, Johannesburg, Forced Migration Studies Programme, $\mathrm{n}^{\circ} 25$, mars 2006.

22. Southern African Migration Project, 2003. Résultats publiés dans Training to Leave : Students and the Brain Drain in Southern Africa, SAMP Migration Policy Series No 35, Kingston and Cape Town, 2005.

23. L. van der Westhuizen, Migration of Skills in South Africa, Patterns, Trends and Challenges, Immigration Advisory Board, 2005; Conférence des Nations unies sur le commerce et le développement, op. cit., p. 5.

24. E. Preston-White et C. Rogerson (dir.), South Africa's Informal Economy, Le Cap, Oxford University Press, 1990.

25. C. Rogerson, "African Immigrant Entrepreneurs and Johannesburg's Changing Inner City », Africa Insight, Pretoria, Africa Institute of South Africa, vol. 27, $\mathrm{n}^{\circ} 4$, 1997; S. Peberdy et C. Rogerson, «Enclave Entrepreneurs? Non-South African Entrepreneurs in South Africa's Informal Sector and Small and Medium Enterprises », Institute of Migration and Ethnic Studies, University of Amsterdam, Netherlands, 1999; M. Reitzes et S. Bam, "Citizenship, Immigration, and Identity in Winterveld, South Africa ", Canadian Journal of African Studies, vol. 34, $\mathrm{n}^{\circ} 1,2000$, pp. 80-100.

26. D. McDonald et al., "Challenging Xenophobia: Myths and Realities About Cross-Border Migration in Southern Africa ", Migration Series, Queens University, Kingston, Southern African Migration Project, n 7, 1998 ; D. McDonald, L. Mashike et C. Golden, «The Lives and Times of African Migrants and Immigrants in Post-Apartheid South Africa ", Migration Series, Queens University, Kingston, Southern African Migration Project, $n^{\circ}$ 13, 1999.

27. M. Reitzes, "Counting the Uncountable ? Undocumented Migrants in South Africa ", Pretoria, Department of Labour, 1998 ; J. Crush, art. cit., 1999; "South Africa : Immigrant numbers a misperception. New Report», UN Integrated Regional Information Network (IRIN), 13 octobre 2005, http://www.irinnews.org/report.asp?ReportID=49540.

28. Republic of South Africa, White Paper on International Migration, Pretoria, Government Printers, 1999.

29. Chiffres annoncés par Busisiwe Mkhewebane-Tshehla, directrice pour les réfugiés, ministère de l'Intérieur sud-africain, agence de presse Mercury, 24 novembre 2005. Ces chiffres sont cependant sujets à caution en raison de la perte d'archives sur des périodes de six mois dans certains bureaux d'enregistrement des réfugiés et des enregistrements multiples de demandeurs d'asile déboutés. Le ministère de l'Intérieur sud-africain espère que l'informatisation prochaine des demandes d'asile et la remise d'une "smart card », carte à puce électronique, permettront d'enrayer la fraude et d'avoir une idée plus précise du nombre total de demandes enregistrées. 
30. Department of Home Affairs, Annual Reports, 1992-1999.

31. Department of Home Affairs, Annual Reports, 1990-2004.

32. J. Handmaker, N. Johnston et J. Schneider, The Status "Regularisation" Programme for Former Mozambican Refugees in South Africa, Johannesburg, Lawyers for Human Rights/University of the Witwatersrand Research Programme, 2001.

33. Department of Home Affairs, Annual Reports, 1993-2004.

34. A. Wa Kabwe-Segatti, « Du rapatriement volontaire au refoulement dissimulé : les réfugiés mozambicains en Afrique du Sud », Politique Africaine, $\mathrm{n}^{\circ}$ 85, mars 2002.

35. Human Rights Watch, «Prohibited Persons » Abuse of Undocumented Migrants, Asylum Seekers and Refugees in South Africa, New York, Human Rights Watch, 1998; South African Human Rights Commission, Report into the Arrest and Detention of Suspected Undocumented Migrants, Parktown, Johannesburg, 19 mars 1999 ; D. McDonald et al., art. cit. ; D. McDonald, L. Mashike et C. Golden, art. cit.

36. D. McDonald (dir.), On Borders: Perspectives on International Migration in Southern Africa, New York, Southern African Migration Project, StMartin's Press, 2000 ; B. Amisi et R. Ballard, «In the Absence of Citizenship : Congolese Refugee and Organisation in South Africa ", Forced Migration Working Paper Series, Johannesburg, Forced Migration Studies Programme, nº 16, avril 2005.

37. S. Lubkemann, "The Transformation of Transnationality among Mozambican Migrants in South Africa", Canadian Journal of African Studies, vol.34, n 1, 2000, pp.41-63; T. Polzer, "Adapting to Changing Legal Frameworks: Mozambicans Refugees in South Africa », Forced Migration Working Paper Series, Johannesburg, Forced Migration Studies Programme, $\mathrm{n}^{\circ} 17$, mai 2005 ; F. Golooba-Mutebi, « Witchcraft, Trust and Reciprocity Among Mozambican Refugees and their South African Hosts in a Lowveld Village ", Forced Migration Working Paper Series, Johannesburg, Forced Migration Studies Programme, ${ }^{\circ}$ 9, juin 2004.

38. L. Van der Westhuizen, op.cit., p. 24.

39. M. Beine, F. Docquier et H. Rapoport, «Brain Drain and LDCs' Growth : Winners and Losers ", Institut zur Zukunft der Arbeit (IZA) Discussion Paper $n^{\circ} 819$, juillet 2003. Disponible sur SSRN : http://ssrn.com/abstract=434542, consulté le 27 mars 2006; W. Blankley, «Brain Circulation - A Work--able Answer to the Brain Drain? », Rapport présenté lors d'une consultation publique du Conseil de l'immigration sud-africain, HSRC, Pretoria, 2003 ; Human Sciences Research Council, «Flight of the Flamingos », rapport pour le Conseil national de l'innovation sud-africain, Pretoria, HSRC Press, 2004.

40. L. Van der Westhuizen, op. cit., p. 25.

41. Republic of South Africa, Immigration Act, 2002 (Act No 13 of 2002).

42. Republic of South Africa, White Paper on International Migration, Pretoria, Government Printers, 1999, p. 30.

43. Republic of South Africa, Immigration Amendment Act, 2004 (Act No 19 of 2004).

44. L. Ensor, « Relief in the Cards for Skilled Expats », Business Day, 24 février 2005.

45. Compagnie de transit créée en 1947, particulièrement active sous les campagnes d'immigration blanche des années 1960-1980.

46. L. Van der Westhuizen, op. cit., p. 18 ; voir également http://www.homecomingrevolution. co.za, site officiel de la campagne.

47. D. MacDonald et J. Crush (éds), Destinations Unknown. Perspectives on the Brain Drain in Southern Africa, Pretoria, Africa Institute of South Africa, Southern African Migration Project, 2002, p. 13.

48. Ibid., traduction de l'auteur.

49. Government Communication and Information Services, 9 octobre 1998.

50. Essop Pahad, ministre de la Présidence, in Barry Streek, "Significant Steps towards Free Movement of Labour in SADC Region », The Mail \& Guardian, 9-15 novembre 2001.

51. Présentation au Centre for Policy Studies, Johannesburg, octobre 2005. 
52. P. Poutignat et J. Streiff-Fénart, «De l'aventurier au commerçant transnational : trajectoires de migrants sub-sahariens en transit à Nouadhibou (Mauritanie)", communication au colloque du Cédej, Circulations migratoires et reconfigurations territoriales entre l'Afrique noire et l'Afrique du Nord, Le Caire, 17-18 novembre 2005.

53. J. Crush, "Northern Gateway: Cross-Border Migration between Namibia and Angola", Migration Series, Kingston, Queens University, Southern African Migration Project, nº 38, 2005.

54. L. Landau, «Transplants and Transients : Nativism, Nationalism and Migration in Inner-City Johannesburg ", Forced Migration Working Paper Series, Johannesburg, Forced Migration Studies Programme, $\mathrm{n}^{\circ} 19$, juillet 2005, p. 17.

55. Il n'existe pas aujourd'hui de statistiques cumulées par nationalité des étrangers résidant en Afrique du Sud, en Namibie et au Botswana. En revanche, il est possible de connaître l'état de la population étrangère dans ces trois pays à travers les recensements de 2001 et les mouvements transfrontaliers annuels, ainsi que le nombre de réfugiés par nationalité d'origine à travers les chiffres du Haut Commissariat aux réfugiés des Nations unies.

56. University of the Witwatersrand - Tufts University Survey, 2003, in C. Kihato et L. Landau, op. cit., 2006, p. 9.

57. Rappelons que des épisodes violents de xénophobie, à travers des manifestations antiimmigrés ou des attaques physiques directes qui ont provoqué la mort d'au moins sept personnes depuis 1994, entachent régulièrement les déclarations de principe des élites politiques encourageant les Sud-Africains à plus de tolérance.

58. L. Landau, op. cit., 2005, p. 16.

\section{RÉSUMÉS}

En retraçant les transformations majeures du cadre migratoire dans l'Afrique australe postapartheid, cet article propose de situer les enjeux économiques qui structurent aujourd'hui la réflexion sur la dynamique migration-développement, afin de mieux comprendre les choix politiques qui contraignent les gouvernements de ces nouveaux eldorados que sont devenus l'Afrique du Sud, le Botswana et la Namibie. Terre d'exil, de transit et d'installation, la pointe sud de l'Afrique australe prend aujourd'hui pleinement conscience des défis liés à son entrée dans le monde. Face à un cosmopolitisme tour à tour revendiqué et honni, les gouvernements de ces trois pays progressent, selon des modalités différenciées, dans la voie de constructions nationales qui ne pourront faire l'économie d'une réflexion plus large sur l'étranger.

In retracing the major changes in migration in post-apartheid Southern Africa, this paper aims to position the economic issues that currently structure discussion on the migration-development dynamics, in order to better understand the political choices constraining the governments of South Africa, Botswana and Namibia, the new lands of opportunity. The tip of Southern Africa - a place of exile, transit and settlement - is now becoming fully aware of the challenges linked to its entry into the world. Confronted by a cosmopolitanism which is alternately claimed and hated, the governments of these three countries are following, under different patterns, nation-building paths that cannot overlook serious reflection on the world at large. 
INDEX

Index géographique : Afrique du Sud, Namibie, Botswana, Afrique australe

Mots-clés : migration

Keywords : South Africa, Botswana, Namibia, Southern Africa, migration

\section{AUTEUR}

\section{AURELIA WA KABWE-SEGATTI}

Normalienne, agrégée d'anglais et docteur en civilisation du monde anglophone et en science politique, Aurelia Wa Kabwe-Segatti est directrice de l'Institut français d'Afrique du Sud à Johannesburg depuis 2004. Elle est l'auteur d'une thèse consacrée à la réforme de la politique d'immigration sud-africaine entre 1986 et 2002. Elle a codirigé avec Philippe Guillaume et Nicolas Péjout L'Afrique du Sud dix ans après Transition accomplie? (Paris, Karthala, 2004). Elle coordonne actuellement avec Loren Landau, de l'université du Witwatersrand, un programme de recherche sur " Immigration, transit et transformation urbaine dans les villes d'Afrique australe » dans le cadre d'un FSP Recherche du ministère des Affaires étrangères.

aurelia@ifas.org.za 